\section{INFEZIONE SISTEMICA DA BARTONELLA HENSELAE ASSOCIATA A PARVOVIRUS B I 9 IN UN SOGGETTO IMMUNOCOMPETENTE IN ETÀ PEDIATRICA.}

Pecorari M., Fabio G., Gennari W., Sabbatini A.T., Nanni N., Govi V., Guaraldi N., Lasagni A., Bagni A., Bertoli G., Casolari C., Portolani M.

Dipartimento Integrato dei Servizi Diagnostici e di Laboratorio, Azienda Ospedaliero-Universitaria di Modena.

(Dipartimento Integrato Materno-Infantile,

Azienda Ospedaliero-Universitaria di Modena).

Introduzione. Bartonella henselae è un parassita degli endoteli ematici e linfatici e dei macrofagi, in grado di provocare infezioni sistemiche pressochè esclusivamente nei soggetti immunocompromessi. Coinfezioni con Parvovirus B19 non sono mai state segnalate in letteratura. Viene riportato un caso inusuale caratterizzato da eritema nodoso e lesioni epatiche granulomatose in una bambina di 5 anni, immunocompetente, nel quale è stato documentato il contemporaneo coinvolgimento di entrambi gli agenti.

Caso clinico. La bambina veniva ricoverata in ambiente ospedaliero per la presenza di noduli sottocutanei eritematosi e dolenti alla superficie estensoria degli arti inferiori e modesti dolori addominali. All'E.O. era apprezzabile una modesta epatomegalia in assenza di linfoadenomegalia e altre obiettività. L'ecografia addominale mostrava una struttura disomogenea in entrambi i lobi per la presenza di multiple lesioni ipoecogene di dimensioni variabili da $0.5 \mathrm{~cm}$ a 1.8 $\mathrm{cm}$. Vie biliari, milza,pancreas, reni e vasi erano nella norma. Gli esami ematologici evidenziavano VES e PCR elevate mentre tutti gli altri parametri risultavano normali. Nell'ipotesi di epatite granulomatosa venivano intraprese ricerche per identificarne l'origine.

Risultati. Indagini anticorpali su siero dimostravano titoli elevati per B.henselae e Parvovirus B19. L'esame istologico su agobiopsia epatica denotava la presenza di infiltrati flogi- stici linfomonocitari. Indagini molecolari in PCR effettuate sul campione bioptico risultavano positive sia per B. henselae che per Parvovirus B19. Il trattamento antibiotico con un macrolide portava alla risoluzione del quadro.

Conclusioni. Non è chiaro il ruolo giocato dall'agente virale nel determinare il quadro clinico anche se è ipotizzabile, da rare segnalazioni, una sua correlazione con le lesioni cutanee. La bambina tuttavia non ha mai presentato le manifestazioni più comuni rapportabili al virus, come esantema similrubeolico o alterazione dell'eritropoiesi. Lesioni epatiche granulomatose, finora mai correlate a Parvovirus B19, sono state se pur raramente associate a infezione da B.henselae in soggetti immunocompetenti. Il ruolo del microrganismo in questo tipo di lesioni è probabilmente sottostimato, particolarmente in età infantile. Tests molecolari in PCR possono rappresentare un valido supporto diagnostico.

Lesioni angiomatose cutanee e peliosi epatica rappresentano evenienze cliniche dell'infezione da B.henselae solitamente diagnosticate nell'ospite immunocompromesso. Dati sperimentali dimostrano tuttavia la capacità di B.henselae a produrre lesioni epatiche granulomatose negli animali senza deficit immunitari. Nei pazienti immunocompetenti con lesioni epato-spleniche, il ruolo di B.henselae è probabilmente sottostimato, particolarmente in età infantile. Come nel nostro caso, non sempre il contatto con i gatti è evidente nella patogenesi dei quadri clinici. 